Pacific Journal of Mathematic 


\title{
LENGTH AND AREA OF A CONVEX CURVE UNDER AFFINE TRANSFORMATION
}

\author{
JOHN W. GREEN
}

1. Introduction. We consider in the plane the class of all convex curves into which a given convex curve can be affinely transformed, and seek the minimum of $L^{2} / A$, where $L$ denotes perimeter and $A$ the area. This amounts to finding the minimum length for a fixed area, or, what is the same thing, to finding the minimum length under area-preserving affine transformations. In $\$ 2$ are found necessary conditions on the supporting function that a given curve yield the minimum of $L^{2} / A$, and in $\S 3$ these are shown to be sufficient. In $\$ 4$ is derived a property of the minimizing curves; namely that if they are sufficiently smooth, they have at least six vertices. In $\$ 5$ is derived an integral representation of the supporting function of a convex curve, and another lemma to be used in $\S 6$. In 6 we study the problem of finding the maximum, over all convex curves, of the minimum over affine transformations of $L^{2} / A$; in other words, we seek that curve of given area, which when affinely transformed so as to minimize its length, gives the greatest length. We show that the extreme curve is a polygon of not more than five sides, but fail to show what is extremely likely, that the solution is a triangle.

For general facts about convex figures and their supporting functions which are used, see [3].

2. Necessary conditions. Consider a convex curve $K$ and its area-preserving affine transforms. Since rigid motions can be ignored, any transformation in which we are interested can be written in the form

$$
T:\left\{\begin{array}{l}
x=e^{\lambda} x^{\prime}, \\
y=\mu x^{\prime}+e^{-\lambda} y^{\prime} .
\end{array}\right.
$$

The length $L(\lambda, \mu)$ of the transformed curve $K(\lambda, \mu)$ is a continuous function of $\lambda$ and $\mu$, and tends to $\infty$ as $\left(\lambda^{2}+\mu^{2}\right)^{1 / 2}$ becomes large. Thus $L(\lambda, \mu)$ has a minimum value, which we take for the moment to be at $\lambda=\mu=0$.

Received June 2, 1952.

Pacific J. Math. 3 (1953), 393-402 
In order to find $L(\lambda, \mu)$ we need the supporting function $p(\lambda, \mu ; \theta)$ of $K(\lambda, \mu)$. If $p(\theta)=p(0,0, \theta)$ is the supporting function of $K$, then a supporting line to $K$ is

$$
x \cos \theta+y \sin \theta=p(\theta) \text {. }
$$

The transformation (1) carries (2) into

$$
x^{\prime}\left(e^{\lambda} \cos \theta+\mu \sin \theta\right)+y^{\prime} e^{-\lambda} \sin \theta=p(\theta)
$$

which is a supporting line to $K(\lambda, \mu)$.

To convert ( 3 ) into normal form we set

$$
\left\{\begin{aligned}
e^{\lambda} \cos \theta+\mu \sin \theta & =k \cos \phi \\
e^{-\lambda} \sin \theta & =k \sin \phi
\end{aligned}\right.
$$

or

$$
\begin{aligned}
\cot \phi & =e^{2 \lambda} \cot \theta+\mu e^{\lambda}, \\
k^{2} & =\left(e^{\lambda} \cos \theta+\mu \sin \theta\right)^{2}+e^{-2 \lambda} \sin ^{2} \theta .
\end{aligned}
$$

The normal form of (3) is then

$$
x^{\prime} \cos \phi+y^{\prime} \sin \phi=p(\theta) / k \text {, }
$$

and so

$$
p(\lambda, \mu, \phi)=p(\theta) / k \text {. }
$$

From ( 5 ) and (4) we see that

$$
\csc ^{2} \phi d \phi=e^{2 \lambda} \csc ^{2} \theta d \theta, e^{2 \lambda} k^{2} \sin ^{2} \phi=\sin ^{2} \theta,
$$

and so $d \phi=d \theta / k^{2}$. Thus ${ }^{1}$

$$
L(\lambda, \mu)=\int p(\lambda, \mu, \phi) d \phi=\int p(\theta) \frac{d \theta}{k^{3}} .
$$

Now let $\lambda$ and $\mu$ be functions of a parameter $t$, with $\lambda(0)=\mu(0)=0$. Then

$$
L(\lambda(t), \mu(t))=L(t)
$$

and direct computation from (6) results in

${ }^{1}$ All integrals go from 0 to $2 \pi$ unless otherwise noted. 


$$
\frac{-L^{\prime}(0)}{3}=\int p(\theta)\left\{\lambda_{0}^{\prime} \cos 2 \theta+\frac{1}{2} \mu_{0}^{\prime} \sin 2 \theta\right\} d \theta=0
$$

Since $\lambda_{0}^{\prime}$ and $\mu_{0}^{\prime}$ may be taken at pleasure, it is clear that in order for $t=0$ to yield a minimum, we must have

$$
\int p(\theta) \cos 2 \theta d \theta=\int p(\theta) \sin 2 \theta d \theta=0 .
$$

In other words, a necessary condition that $K$ give a minimum length is that the sec ond Fourier coefficients of $p$ be zero.

3. Sufficiency. Suppose now that $\lambda=\mu=0$ is a critical value of $L(\lambda, \mu)$, not necessarily the minimum. Then, as in $\S 2$, we see that

$$
\int p \cos 2 \theta d \theta=\int p \sin 2 \theta d \theta=0 .
$$

Futher differentiation of (6), with the use of (8) and certain trigonometric identities, results in

(9) $L^{\prime \prime}(0)=\frac{3}{2} \int p(\theta)\left\{x^{2}(1+5 \cos 4 \theta)+10 x y \sin 4 \theta+y^{2}(1-5 \cos 4 \theta)\right\} d \theta$, where $x=\lambda_{0}^{\prime}, 2 y=\mu_{0}^{\prime}$. Setting

$$
K(\theta)=x^{2}\left(1-\frac{1}{3} \cos 4 \theta\right)-\frac{2}{3} x y \sin 4 \theta+y^{2}\left(1+\frac{1}{3} \cos 4 \theta\right),
$$

we may rewrite (9) as

$$
L^{\prime \prime}(0)=\frac{3}{2} \int p(\theta)\left\{K+K^{\prime \prime}\right\} d \theta \text {. }
$$

Suppose now that $p$ is twice differentiable, and integrate the $K^{\prime \prime}$ term in (11) by parts twice. We get

$$
L^{\prime \prime}(0)=\frac{3}{2} \int\left(p+p^{\prime \prime}\right) K d \theta
$$

The discriminant of the quadratic form (10) is equal to $-32 / 9$, and the form is positive definite. Let $M$ be its minimum value for $x^{2}+y^{2}=1$, and all $\theta$. The quantity $p+p^{\prime \prime}$ is the radius of curvature, $d s / d \theta$, of $K$, and so

$$
L^{\prime \prime}(0) \geq \frac{3}{2} \int M d s=\frac{3}{2} M L
$$


If $p$ is not twice differentiable, we approximate it uniformly by supporting functions which are. The right member of (9), for these approximating functions, is at least $3 M L / 2$, where $L$ is computed for the approximating function; thus, passing to the limit, we see that $(13)$ is satisfied in this case also.

Because of (13), we now see that if $\lambda=\mu=0$ is a critical point for $L(\lambda, \mu)$, then it is a proper relative minimum. Consider now any transformation $T_{0}$, corresponding to parameters $\lambda_{0}, \mu_{0}$, which yields a

$$
K_{0}=K\left(\lambda_{0}, \mu_{0}\right)
$$

for which the second Fourier coefficients of the supporting function vanish. We may write $T$ in the form $\left(T T_{0}^{-1}\right) T_{0}$; that is, in studying the length of the transforms of $K$ as function of $T$, we may study instead the length of the transforms of $K_{0}$ as function of $T T_{0}^{-1}$. We may write

$$
T T_{0}^{-1}:\left\{\begin{array}{l}
x=e^{\left(\lambda-\lambda_{0}\right)} x^{\prime} \\
y=\left(\mu e^{-\lambda_{0}}-\mu_{0} e^{-\lambda}\right) x^{\prime}+e^{-\left(\lambda-\lambda_{0}\right) y^{\prime}}=\eta x^{\prime}+e^{-\xi} y^{\prime},
\end{array}\right.
$$

where

$$
\left\{\begin{array}{l}
\xi=\lambda-\lambda_{0} \\
\eta=\mu e^{-\lambda_{0}}-\mu_{0} e^{-\lambda}
\end{array}\right.
$$

Now

$$
L(\lambda, \mu)=\Omega(\xi, \eta),
$$

and, by the foregoing analysis, $\{(\xi, \eta)$ has a proper relative minimum at $\xi=\eta=$ 0 . But the transformation (14) is nonsingular, and so $L(\lambda, \mu)$ has a proper relative minimum at $\lambda_{0}, \mu_{0}$. Thus every critical point of $L(\lambda, \mu)$ is a proper relative minimum. But an (analytic) function in the plane which has only minima for critical points and which tends to $\infty$ at great distance can have only one critical point [6]. Thus $L(\lambda, \mu)$ has only one critical point, and this must be at the minimum.

THEOREM 1. A necessary and sufficient condition that $K$ have the least length of all curves into which it can be transformed by an area-preserving affine transformation is that

$$
\int p \cos 2 \theta d \theta=\int p \sin 2 \theta d \theta=0 .
$$

Henceforth we shall refer to such $K$ as extreme curves. 
4. A six-vertex theorem. A vertex on a convex curve is a point where the radius of curvature has an extremum. It is a theorem of Kneser ( see for example $[1, \mathrm{p} .160])$ that every convex curve, if sufficiently smooth, has at least four vertices.

THEOREM 2. Each extreme curve with a continuous radius of curvature has at least six vertices. ${ }^{2}$

The radius of curvature $\rho$ is given in terms of the supporting function by $\rho=p+p "$. Now

$$
\int \rho \cos \theta d \theta=\int \frac{d s}{d \theta} \cos \theta d \theta=\int \cos \theta d s=\oint d y=0,
$$

and similarly for $\int \rho \sin \theta d \theta$. Also

$$
\int \rho \cos 2 \theta d \theta=\int\left(p+p^{\prime \prime}\right) \cos 2 \theta d \theta=0,
$$

by two integrations by parts. Thus we see that

$$
\rho \sim \frac{L}{2 \pi}+\sum_{3}^{\infty}\left(a_{n} \cos n \theta+b_{n} \sin n \theta\right)
$$

It has been known since Liouville ( $[5, \mathrm{p} .264])$ that (15) implies that $\rho-L / 2 \pi$ has at least six alternations in signs, and hence $\rho$ six extrema.

In a very similar manner one can prove the following theorem, which however, will only be stated.

THEOREM 3. Each extreme curve intersects a certain circle, of radius $L / 2 \pi$, at least six times.

5. Some lemmas. If $I I(\xi, \mu)$ is the Minkowski Stützfunktion of a convex curve, then

$$
p(\theta)=H(\cos \theta, \sin \theta)
$$

Now $H$ is a convex function of $\xi, \eta ; p(\theta)$ is not convex, but has the somewhat

\footnotetext{
${ }^{2}$ Blaschke [2] has already shown that a convex curve $K$ may be affinely transformed until its radius of curvature is in the form (15), and thus that it has six vertices. However, the vanishing of the coefficients $a_{2}$ and $b_{2}$ was attained in an entirely different way. Namely, he found that ellipse $K_{1}$, of area equal to that of $K$, whose mixed volume with $K$ is a minimum. Transforming affinely so that $K_{1}$ becomes a circle, we see that $K$ becomes a curve satisfying (15). We have not been able to discover that Blaschke or others made any application of this result to the present problem.
} 
analogous property of being sub-sine. A function $f(\theta)$ is sub-sine if, provided

$$
f(\theta)=A \cos \theta+B \sin \theta \text { at } \theta_{1} \text { and } \theta_{2} \text {, where } \theta_{1}<\theta_{2}<\theta_{1}+\pi,
$$

then

$$
f(\theta) \leq A \cos \theta+B \sin \theta \text { for } \theta_{1} \leq \theta \leq \theta_{2} .
$$

A necessary and sufficient condition [4] that a periodic function $p(\theta)$ be the supporting function of a convex curve is that it be sub-sine, or, if it is of class $C^{\prime \prime}$, that $p+p " \geq 0$.

Lemma 1. A necessary and sufficient condition that a function $p(\theta)$ of period $2 \pi$ be the supporting function of a convex curve is that it be expressible in the form

$$
p(\theta)=\int_{\theta_{0}}^{\theta} \sin (\theta-t) d \alpha(t)+A \cos \theta+B \sin \theta
$$

where $\alpha$ is a nondecreasing function.

First let a supporting function $p \subset C^{\prime \prime}$; then

$$
p+p^{\prime \prime}=g(\theta) \geq 0 .
$$

The solution of the differential equation $p+p^{\prime \prime}=g(\theta)$ is readily verified to be

$$
\begin{aligned}
p(\theta)=\int_{\theta_{0}}^{\theta} \sin (\theta-t) g(t) d t & +p\left(\theta_{0}\right) \cos \left(\theta-\theta_{0}\right) \\
& +p^{\prime}\left(\theta_{0}\right) \sin \left(\theta-\theta_{0}\right),
\end{aligned}
$$

which is of the form (16) with

$$
\alpha(\theta)=\int_{\theta_{0}}^{\theta} g(t) d t
$$

Note that

$$
\alpha\left(\theta_{0}\right)=0 \text { and } \alpha\left(\theta_{0}+2 \pi\right)=\int\left(p+p^{\prime \prime}\right) d \theta=L .
$$

Now if $p \notin C^{\prime \prime}$, it is the uniform limit of supporting functions $p_{n}$ which are. We put each $p_{n}$ in the representation (17), and apply the Helly selection theorem and the Bray-Helly theorem ( $[7$, p. 29-31] ) to obtain the result immediately. The factors $p_{n}^{\prime}\left(\theta_{0}\right)$ offer no difficulty, since one easily shows that they are 
bounded for all $n$.

The converse is proved similarly. If a periodic $p$ is given by (16), we can approximate $a$ by a sequence of smooth monotone functions $\alpha_{n}$ which give periodic functions $p_{n}$; these $p_{n}$ are sub-sine since they satisfy

$$
p_{n}^{\prime \prime}+p_{n}^{\prime}=\alpha_{n}^{\prime} \geq 0
$$

Again using the Bray-Helly theorem, we have that $p=\lim p_{n}$; that is, $p$ is a limit of sub-sine functions, and so is sub-sine.

LEMMA 2. If $p(\theta)$ is a supporting function, and if there exist at least six disjoint intervals in $0 \leq \theta \leq 2 \pi$, interior to each of which $p$ is not identically of the form $A \cos \theta+B \sin \theta$, then there exists a function $\eta(\theta)$ with the following properties:

(a) $p+\lambda \eta$ is a supporting function for small $|\lambda|$,

(b) $\int \eta d \theta=\int \eta \cos 2 \theta d \theta=\int \eta \sin 2 \theta d \theta=0$,

(c) $\eta \not \equiv A \cos \theta+B \sin \theta$.

Let $I_{j}: a_{j}<\theta<b_{j}, j=1,2, \cdots, 6$, be the disjoint intervals mentioned, and let $p$ be given by (16). We may assume that $\alpha(\theta)$ is continuous at $a_{j}$ and $b_{j}$. Define

$$
\beta_{j}(\theta)=\left\{\begin{array}{l}
\alpha\left(a_{j}\right) \text { for } 0 \leq \theta<a_{j}, \\
\alpha(\theta) \text { for } a_{j} \leq \theta<b_{j}, \\
\alpha\left(b_{j}\right) \text { for } b_{j} \leq \theta \leq 2 \pi
\end{array}\right.
$$

while outside $(0,2 \pi)$ we make $d \beta_{j}$ periodic. Set

$$
\beta=\sum \lambda_{j} \beta_{j}, \text { where }\left|\lambda_{j}\right| \leq 1
$$

Then $\alpha(\theta)+\lambda \beta(\theta)$ is nondecreasing if $|\lambda| \leq 1$, as simple computation reveals. We set

$$
\eta_{j}=\int_{0}^{\theta} \sin (\theta-t) d \beta_{j}(t) \text { and } \eta=\sum \lambda_{j} \eta_{j}
$$

Then $p+\lambda \eta$ is of the form (16), with $\alpha+\lambda \beta$ in place of $\alpha$. In order that $\eta$ have period $2 \pi$, and thus that $(a)$ be satisfied, we demand that

$$
\sum \lambda_{j} \int \sin \theta d \beta_{j}(\theta)=\sum \lambda_{j} \int \cos \theta d \beta_{j}(\theta)=0
$$


To satisfy conditions ( b) of the lemma, we set

$$
\sum \lambda_{j} \int \eta_{j} d \theta=\sum \lambda_{j} \int \eta_{j} \cos 2 \theta d \theta=\sum \lambda_{i} \int \eta_{i} \sin 2 \theta d \theta=0 .
$$

Equations (19) and (20) comprise five homogeneous equations in the six unknowns $\lambda_{j}$. They always have a nontrivial solution, which we employ for the construction of $\beta$. If $\lambda_{k} \neq 0$, then $\eta$ is equal in $I_{k}$ to a nonzero multiple of $p(\theta)$, plus sine and cosine terms, and this by hypothesis is not of the form $A \cos \theta+$ $B \sin \theta$. Thus ( $\mathrm{c}$ ) is satisfied, and the lemma is proved.

6. The minimax problem. We now restrict our attention to extreme curves, and seek the maximum $m$ of $L^{2} / A$. A crude estimate of $m$ can be obtained as follows. If $K$ is any convex curve of area 1 , inscribe in $K$ a triangle $\Delta$ of maximum area, $A(\Delta)$. Then at each vertex of $\Delta, K$ must have a supporting line parallel to the opposite side of $\Delta$, and these three supporting lines form a triangle $\Delta_{1}$. Transform the plane in an area-preserving affine way so that $\Delta$ and $\Delta_{1}$ are carried into. equilateral triangles $\Delta^{\prime}$ and $\Delta_{1}^{\prime}$, and $K$ into $K^{\prime}$. The perimeter $L\left(\Delta^{\prime}\right)$ of $\Delta^{\prime}$ is given by

$$
L\left(\Delta^{\prime}\right)=6 \sqrt{A\left(\Delta^{\prime}\right) / \sqrt{3}}
$$

Then

$$
L\left(K^{\prime}\right) \leq L\left(\Delta_{1}^{\prime}\right)=2 L\left(\Delta^{\prime}\right)=12 \sqrt{A\left(\Delta^{\prime}\right) / \sqrt{3}} \leq 12 / \sqrt[4]{3}
$$

Thus for the transform $K^{\prime}$ of $K$, we have

$$
L^{2} / A \leq 48 \sqrt{3} \text {, and so } m \leq 48 \sqrt{3}
$$

On the other hand, the equilateral triangle gives

$$
L^{2} / A=12 \sqrt{3} \text {, and so } m \geq 12 \sqrt{3} \text {. }
$$

We now normalize our problem by considering extreme curves of length 1 , and try to minimize the area. By the usual compactness argument $([2, \mathrm{p} .62])$, there does exist a minimizing curve $K$. Let $p$ be the supporting function of $K$. Suppose there exists a function $\eta(\theta)$ satisfying conditions ( a), ( b ) of Lemma 2. Consider the area $A(\lambda)$ of the extreme curve, of unit length, whose supporting function is $p+\lambda \eta$. We have 


$$
\begin{aligned}
2 A(\lambda) & =\int\left\{(p+\lambda \eta)^{2}-\left(p^{\prime}+\lambda \eta^{\prime}\right)^{2}\right\} d \theta \\
& =2 A(0)+2 \lambda \int\left(p \eta-p^{\prime} \eta^{\prime}\right) d \theta+\lambda^{2} \int\left(\eta^{2}-\eta^{\prime 2}\right) d \theta
\end{aligned}
$$

Because of the extreme nature of $K$, the term $\int\left(p \eta-p^{\prime} \eta^{\prime}\right) d \theta=0$. Because of conditions (b) of Lemma 2, the Fourier series of $\eta$ will be as follows.

$$
\eta=a_{1} \cos \theta+b_{1} \sin \theta+\sum_{3}^{\infty}\left(a_{j} \cos j \theta+b_{j} \sin j \theta\right),
$$

and by Parseval's relation,

$$
\frac{1}{\pi} \int \eta^{2} d \theta=\left(a_{1}^{2}+b_{1}^{2}\right)+\sum_{3}^{\infty}\left(a_{i}^{2}+b_{i}^{2}\right)
$$

Similarly ( $\eta^{\prime}$ being bounded),

$$
\frac{1}{\pi} \int \eta^{\prime 2} d \theta=\left(a_{1}^{2}+b_{1}^{2}\right)+\sum_{3}^{\infty} j^{2}\left(a_{i}^{2}+b_{i}^{2}\right)
$$

and so

$$
\int\left(\eta^{2}-\eta^{\prime 2}\right) d \theta=\pi \sum_{3}^{\infty}\left(1-j^{2}\right)\left(a_{i}^{2}+b_{i}^{2}\right)
$$

Since $A(\lambda) \geq A(0)$, we see from (21) and (22) that $a_{j}=b_{j}=0$ for $j \geq 2$, so that $\eta \equiv a_{1} \cos \theta+b_{1} \sin \theta$. Thus it is not possible to satisfy (a), (b), and (c) simultaneously.

Now if $K$ is a polygon, $p$ is piecewise of the form $A \cos \theta+B \sin \theta$, and conversely. If $K$ is not a polygon it is clear that one can find as many intervals as desired in each of which $p$ is not of that form, and Lemma 2 applies. Lemma 2 also applies if $K$ is a polygon of six or more sides. Thus it is not possible for $K$ to be other than a polygon of five or fewer sides.

It appears very likely that $K$ is an equilateral triangle and that $m=12 \sqrt{3}$. To eliminate the cases of four and five sides is just a problem in the calculus, but possibly a very difficult one. In these cases there are not enough sides to construct the variations used above, which consist of sliding sides in and out parallel to themselves, so if a variational method is to be used, a different kind of variation, involving changing the angles, must be found. 


\section{REFERENCES}

1. W. Blaschke, Kreis und Kugel, Leipzig, 1916.

2. Eine Minimumaufgabe über Eilinien, Christiaan Huygens, Internationaal Math. Tijdschrift, 2 (1922-23) 150-154.

3. T. Bonnesen und W. Fenchel, Theorie der Konvexen Köper, Ergebnisse der Mathematik und ihrer Grenzgebiete, vol. 3, no.1, Berlin, 1934.

4. J. W. Green, Sets subtending a constant angle on a circle, Duke Math. J. 17 (1950) 263-267.

5. J. Liouville, Sur le developpement des fonctions, etc., J. Math. Pures Appl. 1, (1836), 253-265.

6. M. Morse, The calculus of variations in the large, Colloquium Publications of the Amer. Math. Soc., New York, 1934 .

7. D. V. Widder, The Laplace transform, Princeton University Press, 1941.

University of California, Los Angeles, and the

Institute for Advanced Study. 


\section{PACIFIC JOURNAL OF MATHEMATICS}

\section{EDITORS}

\section{R. U. FOEINSON}

University of California

Berkeley 4, California

E. HewitT

University of Washington

Seattle 5 , Washington
P. P. DILWOR TH

California Institute of Technology

Pasadena 4, California

E. F. BECKENBACH

University of California

Los Angeles 24, California

\section{ASSOCIATE EDITORS}

$\begin{array}{llll}\text { H. BUSEMANN } & \text { P. R. HALMOS } & \text { BØRGE JESSEN } & \text { J. J. STOKER } \\ \text { HERBERT FFDERER } & \text { IIEINZ HOPF } & \text { PAUL LÉVY } & \text { E. G. STRAUS } \\ \text { MARSHALL, IIALI } & \text { R. D. JAMES } & \text { GEORGE PÓLYA } & \text { KÖSAKU YOSIDA }\end{array}$

\section{SPONSORS}

UNIVERSITY OF BRITISH COLUMBIA

CALIFORNIA INSTITUTE OF TECHNOLOGY.

UNIVERSITY OF CAIJIFORNIA, BERKELEY

UNIVERSITY OF CAIJIFORNIA, DAVIS

UNIVERSITY OF CALIFORNIA, LOS ANGELES

UNIVERSITY OF CALIFORNI A, SANTA BARBARA

UNIVERSITY OF NEVADA

OREGON STATE COLLEGE

UNIVERSITY OF OREGON

\author{
UNIVERSITY OF SOU TIERN CALIFORNIA \\ STANFORD RESEARCH INSTITUTE \\ STANFORD UNIVERSITY \\ WASHINGTON STATE COLLEGE \\ UNIVERSITY OF WASHINGTON \\ AMERICAN MATHEMATICAL SOCIETY \\ NATIONAL BUREAU OF STANDARDS, \\ INSTITUTE FOR NUMERICAL ANALYSIS
}

Mathematical papers intended for publication in the Pacific Journal of Mathematics should be typewritten (double spaced), and the author should keep a complete copy. Manuscripts may be sent to any of the editors except Robinson, whose term expires with the completion of the present volume; they might also be sent to M.M. Schiffer, Stanford University, Stanford, California, who is succeeding Robinson. All other communications to the editors should be addressed to the managing editor, E. F. Beckenbach, at the address given above.

Authors are entitled to receive 100 free reprints of their published papers and may obtain additional copies at cost.

The Pacific Journal of Mathematics is published quarterly, in March, June, September, and December. The price per volume (4 numbers) is $\$ 8.00$; single issues, $\$ 2.50$. Special price to individual faculty members of supporting institutions and to individual members of the American Mathematical Society: $\$ 4.00$ per volume; single issues, $\$ 1.25$.

Subscriptions, orders for back numbers, and changes of address should be sent to the publishers, University of California Press, Berkeley 4, California.

Printed at Ann Arbor, Michigan. Entered as second class matter at the Post Office, Berkeley, California.

\section{UNIVERSITY OF CALIFORNIA PRESS • BERKELEY AND LOS ANGELES}




\section{Pacific Journal of Mathematics}

\section{Vol. 3, No. 2 \\ April, 1953}

William George Bade, An operational calculus for operators with spectrum

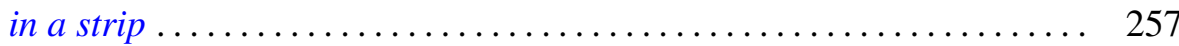

E. F. Beckenbach and Lloyd Kenneth Jackson, Subfunctions of several variables ..................................... 291

David Blackwell, Extension of a renewal theorem ................. 315

L. Carlitz, Some theorems on the Schur derivative ................ 321

Paul Arnold Clement, Generalized convexity and surfaces of negative curvature..................................... 333

Merrill M. Flood, On the Hitchcock distribution problem ............... 369

Watson Bryan Fulks, On the unique determination of solutions of the heat

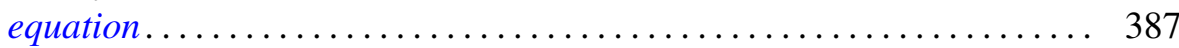

John W. Green, Length and area of a convex curve under affine transformation .................................... 393

William Gustin, An isoperimetric minimax .................. 403

Arthur Eugene Livingston, Some Hausdorff means which exhibit the Gibbs'

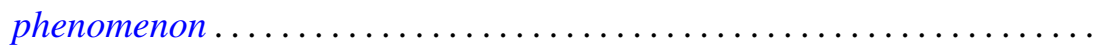

Charles Loewner, On generation of solutions of the biharmonic equation in the plane by conformal mappings ..................... 417

Gábor Szegő, Remark on the preceding paper of Charles Loewner ....... 437

Imanuel Marx and G. Piranian, Lipschitz functions of continuous

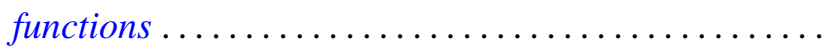

Ting-Kwan Pan, The spherical curvature of a hypersurface in Euclidean space ..

Ruth Lind Potter, On self-adjoint differential equations of second order ...

E. H. Rothe, A note on the Banach spaces of Calkin and Morrey...

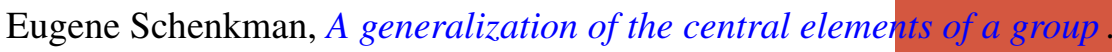

A. Seidenberg, A note on the dimension theory of rings .. . . 